Trauma Berufskrankh 2012 · 14[Suppl 3]:293-298 DOI 10.1007/s10039-011-1818-9

Online publiziert: 17. März 2012

(c) Springer-Verlag 2012

J. Merk

Schule und Abteilung für Physiotherapie, BG-Unfallklinik Tübingen

\title{
Stationäre Rehabilitation nach sprunggelenknahen Frakturen
}

\section{Effekte auf Funktions- und Schmerzparameter sowie die gesundheitsbezogene Lebensqualität}

terbehandlung) bei Patienten mit sprunggelenknahen Frakturen und verzögertem Heilungsverlauf.

In der vorliegenden Arbeit wurden die therapeutischen Effekte einer 3- bis 4-wöchigen stationären Behandlungsmaßnahme bei Patienten mit sprunggelenknahen Frakturen evaluiert. Dabei wurde der Fokus auf Veränderungen in Funktions- parametern (Kraft, Beweglichkeit, Gleichgewicht und Ausdauer), Schmerzen und gesundheitsbezogener Lebensqualität der Patienten gerichtet.

\section{Material und Methode}

Für die vorliegende prospektive Studie wurden Patienten ausgewählt, die sich gung des Patienten und der engen munikation von Arbeitgeber, Berufshelfer bzw. Kostenträger und dem Unfallverletzten kommt sowohl in frühen als auch in späten Nachbehandlungsphasen der physio- und ergotherapeutischen Behandlung eine besondere Bedeutung zu, um verbliebene körperliche Defizite möglichst umfassend auszugleichen bzw. zu vermindern [4].

Wolff [24] stellte an 209 retrospektiv untersuchten Probanden (davon $52 \mathrm{~Pa}-$ tienten mit Sprunggelenk- und Fußverletzungen) fest, dass der Heilungsverlauf von gesetzlich Unfallversicherten gegenüber einem durchschnittlichen Patientenkollektiv verlängert ist [24]. Dabei war bei einem größeren Prozentsatz der arbeitsunfallbedingt am Sprunggelenk verletzten Patienten die berufliche Reintegration nicht zufriedenstellend.

Bislang gibt es keine vergleichenden Untersuchungen zu den Effekten eines komplexen stationären Therapieprogramms im Rahmen einer BGSW (berufsgenossenschaftliche stationäre Wei-

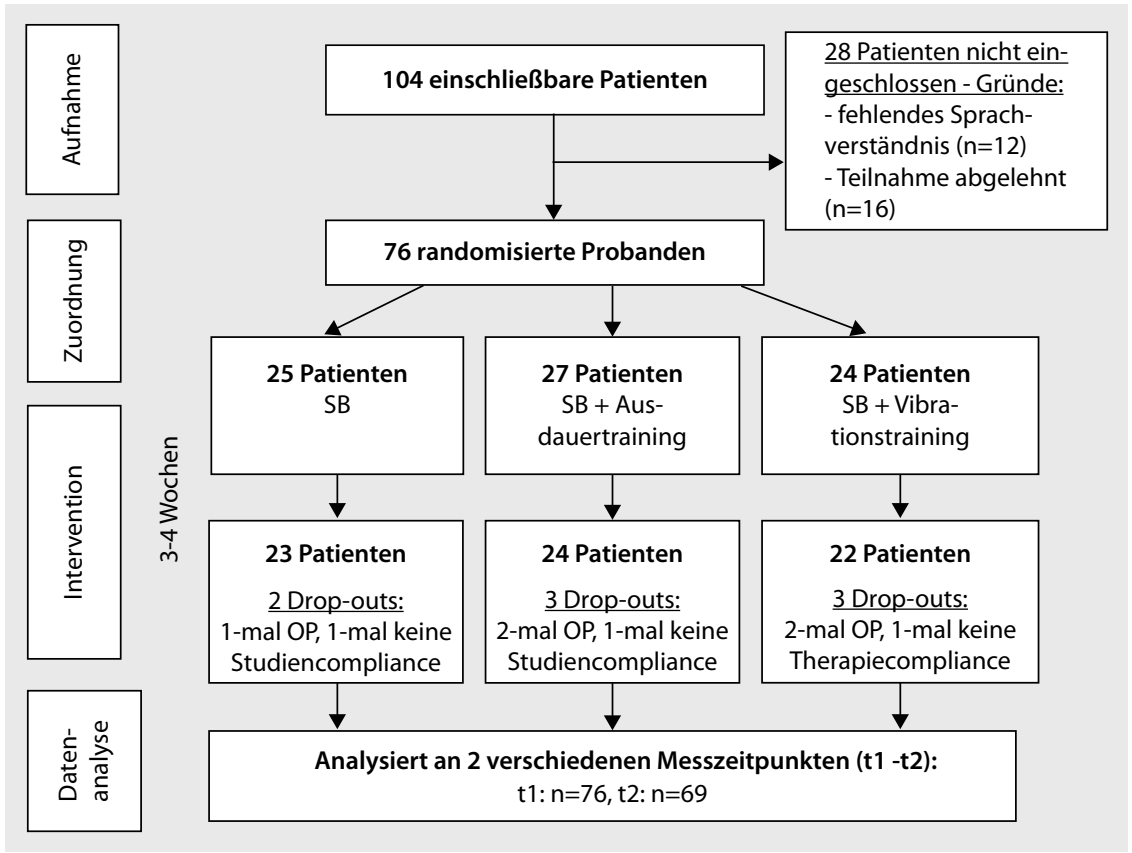

Abb. $1 \Delta$ CONSORT-Schema (CONSORT: , Consolidated standards of reporting trials"): Überblick über Selektion, Randomisierung und Drop-outs der Studienteilnehmer im Verlauf der Untersuchung, OP Operation, SB Standardbehandlung. (Nach [16]) 


\begin{tabular}{|llllll}
\hline \multicolumn{7}{|l}{ Tab. 1 } & Anthropometrische Daten der Probanden & & \\
\hline & \multicolumn{2}{l}{$\begin{array}{l}\text { Standard- } \\
\text { behandlung }\end{array}$} & $\begin{array}{l}\text { Ausdauer- } \\
\text { training }\end{array}$ & $\begin{array}{l}\text { Vibrations- } \\
\text { training }\end{array}$ & $\begin{array}{l}\text { Gesamt bzw. Mittel- } \\
\text { wert bei Alter und BMI }\end{array}$ \\
\hline Anzahl der Patienten & 25 & 27 & 24 & 76 \\
\hline $\begin{array}{l}\text { Ge- } \\
\text { schlecht }\end{array}$ & Männlich & 5 & 4 & 5 & 14 \\
\cline { 2 - 6 } & Weiblich & 20 & 23 & 19 & 62 \\
\hline Alter $($ Jahre) & a & $43,6( \pm 10,9)$ & $42,5( \pm 11,2)$ & $43,4( \pm 10,4)$ & $43,1( \pm 10,7)$ \\
\hline BMI (kg/m²) & $26,3( \pm 4,8)$ & $27,2( \pm 4,8)$ & $26,9( \pm 4,2)$ & $26,8( \pm 4,6)$ \\
\hline $\begin{array}{l}\text { BMl Body-Mass-Index } \\
\text { aMittelwerte, Standardabweichung in Klammern }\end{array}$ & & & \\
\hline
\end{tabular}

Tab. 2 Verletzungsverteilung in den 3 Behandlungsgruppen

\begin{tabular}{|llllll}
\hline Behandlungsgruppe & S & A & V & \\
\hline $\begin{array}{l}\text { Verletzungsverteilung } \\
\text { (absolute Häufigkeiten) }\end{array}$ & $\begin{array}{l}\text { Standard- } \\
\text { behandlung }\end{array}$ & $\begin{array}{l}\text { Ausdauer- } \\
\text { training }\end{array}$ & $\begin{array}{l}\text { Vibrations- } \\
\text { training }\end{array}$ & Gesamt \\
\hline Distale Unterschenkelfraktur & 4 & 7 & 5 & 16 \\
\hline Pilon-tibiale-Fraktur & 2 & 0 & 1 & 3 \\
\hline $\begin{array}{l}\text { Knöchel- } \\
\text { frakturen }\end{array}$ & Weber A & 0 & 2 & 0 & 2 \\
\cline { 2 - 7 } & Weber B & 1 & 0 & 0 & 1 \\
\cline { 2 - 7 } & Weber C & 2 & 2 & 1 & 5 \\
\cline { 2 - 7 } & Innenknöchelfraktur & 1 & 2 & 1 & 4 \\
\cline { 2 - 6 } & Bimalleoläre Fraktur & 2 & 1 & 4 & 7 \\
\cline { 2 - 7 } & Trimalleoläre Fraktur & 2 & 1 & 1 & 4 \\
\hline Talusfraktur & 2 & 7 & 11 & 27 \\
\hline Kalkaneusfraktur & 9 & 7 & & \\
\hline
\end{tabular}

bei einem Arbeitsunfall eine sprunggelenknahe Fraktur zugezogen hatten und aufgrund eines prolongierten Heilungsverlaufs zur BGSW in der Berufsgenossenschaftlichen Unfallklinik (BG-Unfallklinik) Tübingen aufgenommen wurden.

Patienten mit folgenden Monoverletzungen, deren Arbeitsunfall mehr als 3 Monaten zurücklag, wurden in die Studie eingeschlossen:

- distale Unterschenkelfrakturen

- Pilon-tibiale-Frakturen

- Knöchelfrakturen (Typ Weber A, B, C und bi- bzw. trimalleoläre Frakturen, Innenknöchelfrakturen)

- Talusfrakturen

- Kalkaneusfrakturen

Neben oben genannten Primärdiagnosen wurden folgende ergänzende Einschlusskriterien formuliert:

- Einwilligungsfähigkeit der Patienten (insbesondere sprachliches Verständnis)

- Alter 18 bis 55 Jahre

- Beide Geschlechter
Im Zeitraum zwischen Januar 2008 und März 2009 wurden insgesamt 104 Patienten, welche die genannten Einschlusskriterien erfüllten, in die BG-Unfallklinik Tübingen zum stationären Heilverfahren [BGSW/KSR (KSR: komplexe stationäre Rehabilitationsbehandlung)] aufgenommen. Davon gaben 76 (73,1\%; - Tab. 1) ihr schriftliches Einverständnis zur Studienteilnahme und zum Prüfprotokoll der Ethikkommission der Universität Tübingen. Lag die Einverständniserklärung ausgefüllt vor, wurde der Proband nach einer im Vorfeld der Studie erstellten Randomisierungsliste einer der 3 Behandlungsgruppen (Standardbehandlung, Ausdauertraining, Vibrationstraining; 0 Tab. 1, 2) zugeteilt (• Abb. 1).

An beiden Messzeitpunkten, Beginn und Ende der BGSW, wurden mittels Fragebögen die aktuellen Beschwerden [Schmerzintensität nach NRS (numerische Ratingskala)] und die gesundheitsbezogene Lebensqualität (SF-36, [2]) der Patienten erfasst. Außerdem erfolgten Beweglichkeitsmessungen der Sprunggelenke (Neutral-0-Methode mit Goniometer), isokinetische Kraftmessungen der
Plantarflexoren und Dorsalextensoren am CYBEX NORM ${ }^{\mathrm{m}}$ (Firma Cybex, Division of Lumex, Ronkonkoma, New York, USA, Vertrieb durch die Firma Proxomed $\mathrm{GmbH}$, Alzenau) sowie Gleichgewichtstests im 1-Bein-Stand und ein IPN-Ausdauertest $^{\circ}$ (IPN: Institut für Prävention und Nachsorge GmbH, Köln, [10]) auf dem Fahrradergometer.

Alle Probanden absolvierten ein standardisiertes Rehabilitationsprogramm, das aus täglich stattfindender physiotherapeutischer Einzel- und Gruppentherapie, Bewegungsbad, medizinischer Trainingstherapie, Ergotherapie und physikalischen Therapieformen (Elektrotherapie, Eis, Wärme, Wechselbäder) bestand. Die Experimentalgruppe 1 führte zusätzlich ein pulsgesteuertes Ausdauertraining auf dem Fahrradergometer durch. Die Probanden der Versuchsgruppe 2 absolvierten in Ergänzung zum Standardbehandlungsprogramm täglich ein Ganzkörpervibrationstraining auf einem Galileo -Gerät (Modell Galileo Fitness, Firma Novotec Medical, Pforzheim). Eine ausführliche Beschreibung des Therapieprogramms mit differenzierter Erläuterung des progressiven Belastungsaufbaus findet sich bei Merk [13].

\section{Ergebnisse}

Die nachfolgende Darstellung der Ergebnisse bezieht sich der Übersicht wegen auf die gesamte Probandengruppe und wird nicht nach Gruppenunterschieden differenziert.

\section{Gesundheitsbezogene Lebensqualität}

Die SF-36-Fragebogen-Resultate wurden mittels Statistikprogramm SPSS (Version 17.0.1) ausgewertet. Dabei wurden die einzelnen Antwortitems zur Berechnung der Skalen addiert bzw. gewichtet und auf Werte zwischen 0 und 100 transformiert. 0 Tab. 3 zeigt die berechneten Mittelwerte in Relation zur altersgleichen bundesdeutschen gesunden Normgruppe der 5 . Altersdekade (41 bis 50 Jahre). Je höher der dargestellte Wert in der jeweiligen SF-36-Subskala (0-100) ausfällt, desto besser ist die Bewertung dieser Gesundheitskomponente, d. h. ein höherer 
Wert in der Schmerzskala bedeutet eine geringere subjektive Schmerzwahrnehmung. Der Vergleich der Subskalenwerte mit der Referenzgruppe $(n=423)$ ermöglicht eine Beurteilung der Abweichung der ermittelten Messresultate von den zu erwartenden Werten [2].

Am Rehabilitationsbeginn zeigten sich vorwiegend in den körperlichen Gesundheitsdimensionen die größten Normabweichungen. Insbesondere bei der körperlichen Rollenfunktion ergab sich mit einer Differenz von 78,8 Punkten die deutlichsten Differenzen zur Vergleichsgruppe. Aber auch die Dimensionen körperliche Funktionsfähigkeit (-52,7 Punkte) und körperliche Schmerzen (-45,3 Punkte) wiesen große Unterschiede gegenüber der Referenzgruppe auf.

Bei den Befragungen am Ende der stationären Heilbehandlung zeigten sich bis auf die Gesundheitsdimension emotionale Rollenfunktion durchgehend Verbesserungen der Skalenwerte. Die größten $\mathrm{Zu}$ wächse $(28,5-47,3 \%)$ wurden in den 3 körperlichen Gesundheitsskalen erreicht, wobei sich in Relation zur Normgruppe am Ende der BGSW aber auch immer noch die größten Defizite manifestierten.

\section{Schmerzen}

Die Darstellung der Ergebnisse der Befragung zur momentanen und durchschnittlichen Schmerzintensität (Zeitfenster 4 Wochen) nach 11-stufiger numerischer Ratingskala (NRS: 0: kein Schmerz, 10: stärkster vorstellbarer Schmerz) erfolgte analog der SF-36-Resultate in tabellarischer Form (• Tab. 4).

Aus Tab. 4 geht hervor, dass es während der stationären Rehabilitation im Gesamtkollektiv zu leichten Verbesserungen in den beiden erfragten Schmerzintensitäten kam. Die momentane Schmerzstärke reduzierte sich um 14,9\%, die durchschnittliche Schmerzintensität um 10,7\%.

\section{Funktion}

- Tab. 5 gibt die Resultate der Funktionsmessungen an der betroffenen Extremität wieder.

Neben der dargestellten deskriptiven Mittelwertbestimmung wurden bei den oben genannten Messparametern über

Trauma Berufskrankh 2012 · 14[Suppl 3]:293-298 DOI 10.1007/s10039-011-1818-9

(c) Springer-Verlag 2012

J. Merk

\section{Stationäre Rehabilitation nach sprunggelenknahen Frakturen. Effekte auf Funktions- und Schmerzparameter sowie die gesundheitsbezogene Lebensqualität}

\section{Zusammenfassung}

Sprunggelenknahe Frakturen gehören zu den häufigsten Verletzungen nach Berufsunfällen und führen nicht selten zu hohen Behandlungskosten und länger dauernder Arbeitsunfähigkeit. In unserer prospektiven Studie wurden die Effekte eines 3- bis 4-wöchigen, stationär durchgeführten Behandlungsprogramms an 76 Patienten mit sprunggelenknahen Frakturen und verzögertem Heilungsverlauf evaluiert. Zu Beginn und am Ende der stationären Rehabilitation wurden physische Leistungsparameter, die subjektive Lebensgesundheit und die Schmerzen erfasst. Die dabei ermittelten Defizite lassen auf einen großen Rehabilitationsbedarf der untersuchten Patientengruppe schließen. Über den Zeitraum der stationären Behandlung konn- ten der schonungsbedingte Dekonditionierungskreislauf der Patienten durch eine aktivierende Therapie durchbrochen und die Funktionsdefizite signifikant gebessert, wenn auch nicht komplett aufgeholt werden. Das Hauptaugenmerk in der medizinischen Betreuung dieser Patienten sollte der Vermeidung einer Schmerzchronifizierung gelten. Bei einem sich andeutenden verzögerten Heilungsverlauf können durch eine rechtzeitige Einleitung einer BGSW (berufsgenossenschaftliche stationäre Weiterbehandlung) hohe Folgekosten vermieden werden.

\section{Schlüsselwörter}

Sprunggelenk · Fraktur · Arbeitsunfall . Rehabilitation · Therapieeffekte

\section{In-patient rehabilitation treatment for fractures close to the ankle joint. Effects on functional parameters, pain intensity and health-related quality of life}

\section{Abstract}

Fractures close to the ankle joint are the most frequent occupational injuries at the workplace and often lead to high medical costs and long-lasting inability to work. The present prospective study evaluated the effects of in-patient rehabilitation treatment over a period of 3-4 weeks on 76 patients with fractures close to ankle joint and a delayed healing process. Functional parameters were measured at the beginning and at the end of the in-patient rehabilitation treatment. In addition, patients were questioned about pain intensity and health-related quality of life. From the measured deficits it can be concluded that the examined patient groups have a great need for rehabilitation. For the period of in-hospital treatment the decon- ditioning cycle caused by patients attempting to protect the injured limb could be interrupted and the functional restrictions were significantly improved by a predominantly activating therapy, although they could not be completely removed. The main goal of the medical treatment of these patients should be the avoidance of chronic pain. At the first signs of a delayed healing process, high consequential costs due to long periods of work disability can be avoided by beginning in-patient treatment (BGSW - Professional trade association) in a timely manner.

\section{Keywords}

Ankle joint $\cdot$ Fractures, bone $\cdot$ Occupational accidents · Rehabilitation · Therapy effects die SPSS-Prozedur Allgemeines lineares Modell Messwiederholung die Effekte des Faktors Zeit zwischen Messung 1 und 2 ermittelt (Statistikprogramm SPSS, Version 17.0.1): Die induktive Statistik zeigte, dass bei allen Funktions- und Schmerzvariablen signifikante Verbesserungen über den
Zeitraum der stationären Behandlung zu verzeichnen waren $(p<0,05)$.

\section{Diskussion}

Zu den Effekten eines komplex-stationären, standardisierten Behandlungsprogramms bei BG-Patienten mit sprungge- 
Tab. 3 Veränderungen der Mittelwerte in den 8 SF-36-Dimensionen der gesundheitsbezogenen Lebensqualität

\begin{tabular}{|c|c|c|c|c|c|}
\hline \multirow[t]{2}{*}{ SF-36-Skala } & \multicolumn{2}{|c|}{ Befragungszeitpunkt } & \multicolumn{2}{|c|}{ Veränderungen } & \multirow{2}{*}{$\begin{array}{l}\text { Normwertvergleich } \\
\text { (Vergleichsgruppe } 41 \text { bis } \\
50 \text { Jahre) }\end{array}$} \\
\hline & $\begin{array}{l}\text { Beginn der } \\
\text { Rehabilitation }\end{array}$ & $\begin{array}{l}\text { Ende der } \\
\text { Rehabilitation }\end{array}$ & Absolut & Prozentual (\%) & \\
\hline Körperliche Funktionsfähigkeit & 36,21 & 48,21 & $+12,00$ & $+33,1$ & 88,95 \\
\hline Körperliche Rollenfunktion & 8,65 & 12,74 & $+4,09$ & $+47,3$ & 87,49 \\
\hline Körperliche Schmerzen & 33,64 & 43,23 & $+9,59$ & $+28,5$ & 78,90 \\
\hline Allgemeine Gesundheitswahrnehmung & 53,40 & 58,07 & $+4,67$ & $+8,7$ & 68,00 \\
\hline Vitalität & 52,42 & 54,87 & $+2,45$ & $+4,7$ & 64,13 \\
\hline Psychisches Wohlbefinden & 67,08 & 68,23 & $+1,15$ & $+1,7$ & 73,66 \\
\hline Emotionale Rollenfunktion & 64,78 & 55,35 & $-9,43$ & $-14,6$ & 91,51 \\
\hline Soziale Funktionsfähigkeit & 67,69 & 73,13 & $+5,44$ & $+8,0$ & 89,36 \\
\hline
\end{tabular}

Tab. 4 Schmerzintensitätsveränderungen während der stationären Rehabilitation

\begin{tabular}{|c|c|c|c|c|c|c|c|}
\hline & & \multicolumn{3}{|c|}{ Momentane Schmerzstärke } & \multicolumn{3}{|c|}{ Durchschnittliche Schmerzstärke } \\
\hline & & \multicolumn{2}{|l|}{ NRS } & \multirow{2}{*}{$\begin{array}{l}\text { Mittelwert- } \\
\text { differenz }\end{array}$} & \multicolumn{2}{|l|}{ NRS } & \multirow{2}{*}{$\begin{array}{l}\text { Mittel- } \\
\text { wertdiffe- } \\
\text { renz }\end{array}$} \\
\hline & & $\begin{array}{l}\text { 1. Mes- } \\
\text { sung }\end{array}$ & $\begin{array}{l}\text { 2. Mes- } \\
\text { sung }\end{array}$ & & $\begin{array}{l}\text { 1. Mes- } \\
\text { sung }\end{array}$ & $\begin{array}{l}\text { 2. Mes- } \\
\text { sung }\end{array}$ & \\
\hline \multirow{2}{*}{$\begin{array}{l}\text { Gesamt- } \\
\text { gruppe } \\
(n=69)\end{array}$} & M & 3,88 & 3,30 & $-0,58$ & 4,81 & 4,30 & $-0,51$ \\
\hline & SD & $\pm 2,17$ & $\pm 2,18$ & & $\pm 1,96$ & $\pm 1,90$ & \\
\hline
\end{tabular}

M Mittelwert, $\mathrm{n}$ Probandenanzahl, NRS numerische Ratingskala, SD Standardabweichung

lenknaher Fraktur gibt es bislang keine deutschsprachigen Vergleichsstudien. Es existieren zwar einige Untersuchungen zur Effektivität von multimodalen stationären Behandlungsprogrammen bei Rückenschmerzen [19, 21]. Unmittelbare Ergebnisvergleiche zu diesen Studien sind aber deshalb schwierig, weil so gut wie nie Funktionsergebnisse (Kraft, Beweglichkeit, Ausdauer) und häufig nur Schmerzparameter als Outcome-Größen genannt werden.

\section{Schmerz}

Die Analyse der Ergebnisse zur Schmerzbefragung ergaben, dass die Schmerzintensitäten der im vorliegenden Beitrag behandelten Probandengruppe deutlich über denen von Vergleichsstudien lagen [11, 22, 23]. Gründe für die aggravierten Beschwerden sind darin zu sehen, dass die Schmerzangaben von noch ausstehenden finanziellen Aspekten [Rentengutachten, MdE-Festlegung (MdE: Minderung der Erwerbsfähigkeit)] beeinflusst werden $[10,16]$.

Ein weiterer Punkt, der aus der Sicht des Autors Anlass zum Handeln gibt, ist die Tatsache, dass die Studienpatienten bereits chronifizierte Schmerzmuster zeigten: Die mittlere Zeitdauer zwischen Arbeitsunfall und stationärem Rehabilitationsbeginn lag in unserer Probandengruppe bei 189,7 Tagen. Nach einem Zeitraum von mehr als 6 Monaten jedoch sind die primären Heilungsprozesse im geschädigten Gewebe in der Regel abgeschlossen. Der sich bereits chronifizierende Schmerz hat seine biologische Warnfunktion verloren, was zu einem Pathomechanismus mit erhöhter zentraler Sensibilisierung $[3,7]$ führen kann.

Auf die Probanden der vorliegenden Studie treffen sehr viele der bekannten Risikofaktoren für eine Chronifizierung der Beschwerden zu: Ihr Bildungsniveau war niedrig, und alle Patienten erhielten Krankengeldzahlungen. Der körperliche Trainingszustand der Probanden wies eine deutliche Dekonditionierung mit vorhandener Aktivitätsintoleranz auf. Die Ursache hierfür kann neben einem eher inaktiven Alltagsverhalten auch darauf zurückzuführen sein, dass der größte Teil der Patienten ambulant überwiegend passiv behandelt worden war (Medikamente, Massage, Mobilisationstechniken). Zudem fehlte den Probanden aus den vorstationären Therapien häufig die Information, dass gezielte körperliche Aktivität keinen Schaden anrichtet, sondern den Heilungsverlauf günstig beeinflusst. In der Anamnesebefragung entstand teilweise der Eindruck, dass die vor der stationären Behandlung erfolgten Maßnahmen nicht befund- und arbeitsplatzorientiert konzipiert, sondern im Sinne der Kundenbindung als therapeutische Dienstleistung nach dem Wunsch der Patienten angeboten worden waren. Insofern liegt der Wert des stationären Heilverfahrens neben der Verbesserung physischer Leistungsparameter (Rekonditionierung) auch darin, bei den Patienten inadäquate Verhaltensmuster (übertriebene Schonung, Angst vor körperlicher Aktivität) zu verändern [14]. Der standardisierte aktive Therapieansatz an der BG-Unfallklinik Tübingen verwunderte deshalb anfänglich viele der bisher vorwiegend passiv behandelten Studienpatienten. Die ungewohnte körperliche Aktivität führte zunächst auch häufig zu einer Beschwerdeverstärkung. Die Verbesserungen in den Schmerzwerten während der stationären Behandlung zeigten allerdings, dass das Therapieprogramm in einer Reduktion des wahrgenommenen Schmerzes resultierte und bei den Patienten die sehr wichtige Fähigkeit erhöhte, in der körperlichen Aktivität (wieder) den eigenen Schmerz zu tolerieren. Diese nicht zu unterschätzende Wirkung des stationären Heilverfahrens bildet nach Meinung des Autors die Voraussetzung dafür, dass die berufliche Wiedereingliederung in eine körperlich aktive Tätigkeit gelingen kann.

Aus diesen Erkenntnissen kann für die Patientengruppe mit sprunggelenknahen Frakturen abgeleitet werden, dass ein früher Behandlungsbeginn einer Chroni- 


\begin{tabular}{|c|c|c|c|c|c|c|}
\hline \multicolumn{3}{|l|}{ Funktionsparameter } & $\begin{array}{l}\text { 1. Messung (Beginn } \\
\text { der Rehabilitation) }\end{array}$ & $\begin{array}{l}\text { 2. Messung (Ende } \\
\text { der Rehabilitation) }\end{array}$ & $\begin{array}{l}\text { Veränderungen über } \\
\text { den BGSW-Zeitraum }\end{array}$ & $\begin{array}{l}\text { Restdefizit zur } \\
\text { gesunden Seite }\end{array}$ \\
\hline \multirow{3}{*}{$\begin{array}{l}\text { OSG-Beweglichkeit } \\
\text { (Winkelgrade) }\end{array}$} & \multicolumn{2}{|c|}{ Dorsalextension } & 7,75 & 11,38 & $+3,63(+46,8 \%)$ & $-3,62$ \\
\hline & \multicolumn{2}{|c|}{ Plantarflexion } & 22,90 & 26,38 & $+3,48(+15,2 \%)$ & $-6,95$ \\
\hline & \multicolumn{2}{|c|}{ Gesamt } & 30,65 & 37,76 & $+7,11(+23,2 \%)$ & $-10,57$ \\
\hline \multirow{3}{*}{$\begin{array}{l}\text { USG-Beweglichkeit } \\
\text { (Winkelgrade) }\end{array}$} & \multicolumn{2}{|c|}{ Inversion } & 11,96 & 18,04 & $+6,08(+50,8 \%)$ & $-7,03$ \\
\hline & \multicolumn{2}{|c|}{ Eversion } & 5,51 & 9,71 & $+4,20(+76,2 \%)$ & $-2,83$ \\
\hline & \multicolumn{2}{|c|}{ Gesamt } & 17,47 & 27,75 & $+10,28(+58,8 \%)$ & $-9,86$ \\
\hline \multirow{2}{*}{$\begin{array}{l}\text { Gleichgewicht im } \\
\text { 1-Bein-Stand, Zeitdauer } \\
\text { (s) }\end{array}$} & \multicolumn{2}{|c|}{ Augen geöffnet, auf dünner Matte } & 8,97 & 16,75 & $+7,78(+86,7 \%)$ & $-11,74$ \\
\hline & \multicolumn{2}{|c|}{ Augen geschlossen, keine Matte } & 3,23 & 6,07 & $+2,84(+87,9 \%)$ & $-6,74$ \\
\hline \multirow{2}{*}{$\begin{array}{l}\text { Maximalkraft } \\
\text { [Drehmomentmaxima } \\
\text { bei } 30^{\circ} / \mathrm{s} \text { ] }(\mathrm{Nm})\end{array}$} & \multicolumn{2}{|c|}{ Dorsalextensoren } & 18,25 & 23,68 & $+5,43(+29,8 \%)$ & $-5,90$ \\
\hline & \multicolumn{2}{|c|}{ Plantarflexoren } & 40,20 & 67,68 & $+27,48(+68,4 \%)$ & $-46,78$ \\
\hline \multicolumn{3}{|c|}{ Ausdauerleistung/relativer IPN-Wert (W/kgKG) } & 1,2860 & 1,4424 & $+0,1564(+12,2 \%)$ & \\
\hline \multirow{5}{*}{$\begin{array}{l}\text { Ausdauer-/Fitness- } \\
\text { stufen (Häufigkeits- } \\
\text { verteilung, } n=68 \text { ) }\end{array}$} & 1 & $\begin{array}{l}\text { Deutlich unter dem } \\
\text { Durchschnitt }\end{array}$ & $47(69,1 \%)$ & $31(45,6 \%)$ & -16 & \\
\hline & 2 & $\begin{array}{l}\text { Unter dem } \\
\text { Durchschnitt }\end{array}$ & $10(14,7 \%)$ & $16(23,5 \%)$ & +6 & \\
\hline & 3 & Durchschnitt & $10(14,7 \%)$ & $18(26,5 \%)$ & +8 & \\
\hline & 4 & $\begin{array}{l}\text { Über dem } \\
\text { Durchschnitt }\end{array}$ & $0(0,0 \%)$ & $2(2,9 \%)$ & +2 & \\
\hline & 5 & $\begin{array}{l}\text { Deutlich über dem } \\
\text { Durchschnitt }\end{array}$ & $1(1,5 \%)$ & $1(1,5 \%)$ & \pm 0 & \\
\hline
\end{tabular}

BGSW berufsgenossenschaftliche stationäre Weiterbehandlung, IPN Institut für Prävention und Nachsorge GmbH Köln, KG Körpergewicht, OSG oberes Sprunggelenk, USG unteres Sprunggelenk

fizierung der Schmerzproblematik entgegen wirkt [21].

\section{Gesundheitsbezogene Lebensqualität}

Die Resultate der SF-36-Befragung zeigen, dass im vorliegenden Probandengut deutliche Einschränkungen insbesondere der körperlichen Gesundheitsdimensionen auch noch 6 Monate nach dem Arbeitsunfall vorlagen. Die Resultate einiger Studien $[1,18,20]$ lassen erwarten, dass es im Anschluss an eine knöcherne Sprunggelenkverletzung sogar noch nach 12 Monaten zu deutlichen funktionellen Zuwächsen und subjektiven Verbesserungen im Gesundheitszustand kommen kann. Bei den Studienprobanden handelte es sich zudem nicht wie bei den Vergleichsstudien um unkomplizierte Rehabilitationsverläufe, sondern um Patienten, die gerade aufgrund ihres verzögerten Heilungsverlaufs zur intensiven stationären Therapie aufgenommen worden waren.

\section{Funktion}

Sprunggelenkbeweglichkeit. Als therapeutische Konsequenz aus den erfassten Funktionsparametern lässt sich ableiten, dass der Fokus bei der Sprunggelenkbeweglichkeit insbesondere auf die alltagsrelevante Dorsalextension im oberen Sprunggelenk gelegt werden sollte. Dieser Parameter wird von verschiedenen Autoren als Prädiktor für eine erfolgreiche Rehabilitation der Betroffenen angesehen $[12,17]$.

Gleichgewicht. Bei den durchgeführten Koordinationstests wurden sehr große Differenzen zwischen den Zeiten im 1-Bein-Stand mit geöffneten und geschlossenen Augen registriert. Dies zeigt, dass es den Patienten sehr viel schwerer fällt, das Gleichgewicht ohne Sichtkontrolle zu kontrollieren. Aus diesen Erkenntnissen muss für die Therapie abgeleitet werden, die optische Kontrolle bei der Gleichgewichtsschulung bewusst $\mathrm{zu}$ reduzieren, um gezielt propriozeptive
Qualitäten der Tiefensensibilität zu schulen.

Kraft. Bei den Kraftmessungen mittels CYBEX NORM $^{m s}$ fiel auf, dass die Einschränkungen im Maximalkraftbereich insbesondere die Plantarflexionsmuskulatur betreffen. Therapeutische Konsequenz daraus muss sein, dass das medizinische Aufbautraining im Anschluss an eine sprunggelenknahe Fraktur immer schwerpunktmäßig auf die plantarflexorischen Muskeln ausgerichtet sein sollte.

Ausdauer. Die Bestimmung der Grundlagenausdauer hatte zum Ziel, den allgemeinen körperlichen Fitnesszustand der Probanden zu erfassen. Da eine große Mehrzahl der untersuchten Patienten physisch anstrengende Berufe ausübt, kann davon ausgegangen werden, dass ein Mindestmaß an Ausdauerleistungsfähigkeit die Basis für ihre erfolgreiche berufliche Wiedereingliederung darstellt. Unter diesem Blickwinkel können die Ausdauerergebnisse des Großteils der Probanden bei den Eingangsmessun- 
gen auch als auffällig schwach bezeichnet werden. Therapeutisch sollte daraus abgeleitet werden, dass Ausdauertrainingsreizen in der Nachbehandlung von Patienten mit Sprunggelenkfrakturen ein größerer therapeutischer Stellenwert beigemessen werden sollte. Insbesondere in den Phasen der Teilbelastung sollte über nichtgewichtsbelastende körperliche Aktivitäten (Schwimmen, Aquajogging, Fahrradergometertraining) einem massiven $\mathrm{Ab}$ bau der Grundlagenausdauer entgegengewirkt werden. Dem Training der HerzKreislauf-Leistungsfähigkeit in späteren Nachbehandlungsphasen kommt unter Berücksichtigung der Tatsache, dass die Probanden der Ausdauergruppe im vorliegenden Kollektiv durchschnittlich knapp 20 Tage schneller in den Beruf reintegriert werden konnten als die arbeitsfähigen Probanden der anderen Therapiegruppen, eine noch größere Bedeutung zu. Der allgemeine Ausdauertrainingszustand scheint also in der ganzheitlichen Betrachtung der Patienten nach sprung gelenknahen Verletzungen eine bisher unterschätzte Bedeutung zu haben.

\section{Resümee und Ausblick}

Die erfassten Funktions- und Beschwerdeparameter lassen auf einen großen Rehabilitationsbedarf in der untersuchten Patientengruppe schließen. Über den Zeitraum der BGSW gelang es, den schonungsbedingten Dekonditionierungskreislauf der Patienten durch eine schwerpunktmäßig aktivierende Therapie zu durchbrechen. Die manifesten Funktionseinschränkungen konnten signifikant gebessert, wenn auch nicht komplett aufgeholt werden. Die signifikanten Verbesserungen aller Funktions- und Schmerzparameter im Verlauf der stationären Rehabilitation zeigen, dass die BGSW an der BG-Unfallklinik Tübingen ein hocheffektives Rehabilitationsinstrument darstellt. Ein aktiver Therapieansatz zur Rekonditionierung der BGSW-Patienten ist sinnvoll und notwendig, um verlorengegangene Belastungstoleranzen wieder aufzubauen bzw. Schmerzchronifizierungsprozesse zu durchbrechen. In Anbetracht der körperlich hohen beruflichen Anforderungen vieler BGSW-Patienten sollte ein komplex-stationäres Therapieprogramm ein individuelles Ausdauertraining beinhalten.

Optimierungsbedarf besteht aus Sicht des Autors bei der ambulanten Therapie, welche häufig rein passiv-physikalische Methoden beinhaltet, und bei der Beratung der Unfallverletzten, die zu wenig die Eigenverantwortung und Aktivität des Patienten einfordert. Auch wenn bei stetig wachsendem Kostendruck im Gesundheitswesen die komplex-stationären Rehabilitationsbehandlungen immer wieder als kostenintensiv gelten, können durch eine frühzeitige Einleitung eines stationär durchgeführten, multidisziplinären Rehabilitationsprogramms hohe Folgekosten eingespart werden.

\begin{tabular}{l} 
Korrespondenzadresse \\
\hline \\
Dr. J. Merk \\
Schule und Abteilung \\
für Physiotherapie, \\
BG-Unfallklinik Tübingen, \\
Schnarrenbergstraße 95, \\
72076 Tübingen \\
jmerk@bgu-tuebingen.de
\end{tabular}

Interessenkonflikt. Der korrespondierende Autor gibt an, dass kein Interessenkonflikt besteht.

The supplement containing this article is not sponsored by industry.

\section{Literatur}

1. Belcher GL, Radomisli TE, Abate JA, Stabile LA, Trafton PG (1997) Functional outcome analysis of operatively treated malleolar fractures. J Orthop Trauma 11:106-109

2. Bullinger B, Kirchberger I (1998) SF-36 Fragebogen zum Gesundheitszustand, Handanweisung. Hogrefe, Göttingen

3. Butler DS, Moseley L (2005) Schmerzen verstehen Springer, Berlin Heidelberg New York

4. Campbell JT (2002) Foot and ankle fractures in the industrial setting. Foot Ankle Clin 7:323-350

5. Conti SF, Silverman L (2002) Epidemiology of foot and ankle injuries in the workplace. Foot Ankle Clin 7:273-290

6. Eggli D (1987) Maßstab für Kräfte. In: Ow D von, Hüni G (Hrsg) Muskuläre Rehabilitation. Perimed, Erlangen, S 86-98

7. Gifford L (2000) Schmerzphysiologie. In: Berg F van den (Hrsg) Angewandte Physiologie, Bd 2 Organsysteme verstehen und beeinflussen. Thieme, Stuttgart New York, S 467-518

8. Kropf U, Müller WD, Lohsträter A, Bak P, Smolenski UC (2006) Effizienz und Kosteneffektivität der Berufsgenossenschaftlichen Stationären Weiterbehandlung (BGSW) nach Verletzungen der Wirbelsäule und der unteren Extremität. Phys Med Rehabil Kuror 16:160-165
9. Kunkel M, Miller SD (2002) Return to work after foot and ankle injury. Foot Ankle Clin 7:421-427

10. Lagerstrom D, Trunz E (1997) IPN-Ausdauertest. Gesundheitssport Sportther 13:68-71

11. Lin CC, Moseley AM, Herbert RD, Refshauge KM (2009) Pain and dorsiflexion range of motion predict short- and medium-term activity limitation in people receiving physiotherapy intervention after ankle fracture: an observational study. Aust J Physiother 55:31-37

12. Lin CC, Moseley AM, Refshauge KM (2009) Effects of rehabilitation after ankle fracture: a Cochrane systematic review. Eur J Phys Rehabil Med 45:431441

13. Merk J (2011) Rehabilitation bei sprunggelenksnahen Frakturen nach Arbeitsunfall - Stationäre Behandlungseffekte auf Schmerzen, physische Leistungsfaktoren und die gesundheitsbezogene Lebensqualität. Südwestdeutscher Verlag für Hochschulschriften, Saarbrücken

14. Merk J, Fischle G, Pappon R (2007) Stark im Team Modell einer interdisziplinären Schmerztherapie. Physiopraxis 5:22-25

15. Moher D, Schulz KF, Altman DG (2004) Das CONSORT Statement: Überarbeitete Empfehlungen zur Qualitätsverbesserung von Reports randomisierter Studien im Parallel-Design. Dtsch Med Wochenschr 129:T16-T20

16. Neuburger M, Schmelz M (2007) Schmerzchronifizierung - Ursachen und Therapieoptionen. Trauma Berufskrankh 9:103-106

17. Nilsson G, Nyberg P, Ekdahl C, Eneroth M (2003) Performance after surgical treatment of patients with ankle fractures - 14-month follow-up. Physiother Res Int 8: 69-82

18. Obremskey WT, Dirschl DR, Crother JD, Craig WL, Driver RE, LeCroy CM (2002) Change over time of SF-36 functional outcomes for operatively treated unstable ankle fractures. J Orthop Trauma 16:3033

19. Pfingsten M, Hildebrandt J (1999) Neue Wege in der Behandlung chronischer Rückenschmerzen. In: Hoefert HW, Kröner-Herwig B (Hrsg) Schmerzbehandlung - psychologische und medikamentöse Interventionen. Reinhardt, Basel, S 203-223

20. Ponzer S, Nasell H, Bergman B, Tornkvist H (1999) Functional outcome and quality of life in patients with type B ankle fractures: a two-year follow-up study. J Orthop Trauma 13:363-368

21. Rohde J (2002) Schmerzreduktion bei Patienten mit akuten lumbalen Radikulär- und Pseudo-Radikulärsyndromen nach stationärer manueller Therapie und komplexer Physio- und Schmerztherapie. Manuell Med 40:203-209

22. Simanski CJ, Maegele MG, Lefering R, Lehnen DM, Kawel N, Riess P, Yucel N, Tiling T, Bouillon B (2006) Functional treatment and early weightbearing after an ankle fracture: a prospective study. J Orthop Trauma 20:108-114

23. Weening B, Bhandari M (2005) Predictors of functional outcome following transsyndesmotic screw fixation of ankle fractures. J Orthop Trauma 19:102-108

24. Wolff D (2007) Therapeutischer Stellenwert der BGSW bei Verletzungen des Bewegungsapparats. Trauma Berufskrankh 9:143-147 